$\underline{\text { Preprint typeset in JHEP style - PAPER VERSION }}$

PITHA 05/02

PSI-PR-05-03

WUE-ITP-2005-002

\title{
Gluon-induced $W W$ background to Higgs boson searches at the LHC
}

\author{
T. Binoth \\ Institut für Theoretische Physik, Universität Würzburg, D-97074 Würzburg, Germany
}

\author{
M. Ciccolini \\ Paul Scherrer Institut, CH-5232 Villigen PSI, Switzerland
}

\author{
N. Kauer \\ Institut für Theoretische Physik E, RWTH Aachen, D-52056 Aachen, Germany
}

\author{
M. Krämer \\ Institut für Theoretische Physik E, RWTH Aachen, D-52056 Aachen, Germany
}

\begin{abstract}
Vector-boson pair production is an important background for Higgs boson and new physics searches at the Large Hadron Collider LHC. We have calculated the loop-induced gluon-fusion process $g g \rightarrow W W \rightarrow$ leptons, allowing for arbitrary invariant masses of the intermediate $W$ bosons. This process contributes at $\mathcal{O}\left(\alpha_{\mathrm{s}}^{2}\right)$ relative to quarkantiquark annihilation, but its importance is enhanced by the large gluon flux at the LHC and by experimental cuts employed in Higgs boson searches. We find that $g g \rightarrow W W$ provides only a moderate correction (ca. $5 \%$ ) to the inclusive $W$-pair production cross section at the LHC. However, after taking into account realistic experimental cuts, the gluon-fusion process becomes significant and increases the theoretical $W W$ background estimate for Higgs searches in the $p p \rightarrow H \rightarrow W W \rightarrow$ leptons channel by approximately $30 \%$.
\end{abstract}

Keywords: QCD, Higgs Physics, Hadronic Colliders. 


\section{Contents}

1. Introduction 1

2. Calculation 2

3. Results 3

4. Conclusions 6

\section{Introduction}

The search for Higgs particles and for physics beyond the Standard Model (SM) is central to the physics program at the future Large Hadron Collider (LHC) at CERN. One of the most promising processes for Higgs-boson discovery in the mass range $M_{\text {Higgs }}$ between 140 and $180 \mathrm{GeV}$ is the $p p \rightarrow H \rightarrow W^{*} W^{*} \rightarrow \ell \bar{\nu} \bar{\ell}^{\prime} \nu^{\prime}$ channel [1, 2], where $p p \rightarrow H$ proceeds through gluon-gluon fusion. ${ }^{1}$ The dominant irreducible background to this process is $W$ pair continuum production, which has a cross section times branching ratio more than five times larger than the Higgs-boson signal. An accurate theoretical prediction for the background process is crucial to fully exploit the $p p \rightarrow H \rightarrow W^{*} W^{*} \rightarrow \ell \bar{\nu} \bar{\ell}^{\prime} \nu^{\prime}$ discovery channel, in particular as no Higgs mass peak can be reconstructed from leptonic $W$ decays. Besides its significance as a background to Higgs searches, vector-boson pair production is an important testing ground for the non-Abelian structure of the SM in general. It furthermore provides a background for many new physics searches.

In this paper we present a calculation of the gluon-induced contribution $g g \rightarrow W^{*} W^{*} \rightarrow$ $\ell \bar{\nu} \bar{\ell}^{\prime} \nu^{\prime}$ which proceeds through a quark loop as shown in Fig. 1. Although suppressed by two powers of $\alpha_{\mathrm{s}}$ relative to quark-antiquark annihilation, gluon-fusion is a potentially significant source of $W$-boson pairs at the LHC because of the large gluon-gluon luminosity. Furthermore, the importance of gluon-gluon induced background processes is enhanced by the experimental Higgs search cuts which exploit the longitudinal boost and the spin correlations of the $W W$ system to suppress $W$-pair continuum production through quarkantiquark annihilation.

The hadronic production of $W$ pairs has been studied extensively in the literature. A recent review can be found in Ref. [4]. Next-to-leading order (NLO) QCD corrections to $q \bar{q} \rightarrow W W$ have been presented in Refs. [5, 6], while NLO calculations for $q \bar{q} \rightarrow W W \rightarrow$ $\ell \bar{\nu} \bar{\ell}^{\prime} \nu^{\prime}$ including spin and decay angle correlations can be found in Refs. [7, 8, 9, 10]. At large $W W$ invariant masses, also electroweak corrections become important [11]. The

\footnotetext{
${ }^{1}$ Another favourable Higgs discovery channel is the $H \rightarrow W W$ decay mode in weak boson fusion, $p p \rightarrow H(\rightarrow W W)+q q[3]$.
} 
gluon-gluon induced contribution to on-shell $W$-pair production, $g g \rightarrow W W$, has first been calculated in Ref. [12] for the case of massless quarks circulating in the loop. The calculation has been extended in Ref. [13] to include the top-bottom massive quark loop. In this paper we present the first calculation of the gluon-induced process $g g \rightarrow W^{*} W^{*} \rightarrow \ell \bar{\nu} \bar{\ell}^{\prime} \nu^{\prime}$, including spin and decay angle correlations and allowing for arbitrary invariant masses of the intermediate $W$ bosons. The main purpose of the calculation is to study the impact of gluon-gluon induced backgrounds to Higgs boson searches in the $H \rightarrow W^{*} W^{*} \rightarrow \ell \bar{\nu} \bar{\ell}^{\prime} \nu^{\prime}$ decay mode. In a first step, and in order to quantify the general importance of the gluonfusion process, we restrict our calculation to the leading contribution which arises from intermediate light quarks of the first two generations. Moreover, we do not take into account gluon-gluon induced tree-level processes of the type $g g \rightarrow W W q \bar{q}$, which have been found to be strongly suppressed in hadronic $W Z, W \gamma$ and $Z \gamma$ production [14]. We finally note that a calculation similar to ours has been performed for the process $g g \rightarrow Z^{*} Z^{*} \rightarrow 4 l^{ \pm}$in Ref. [15].

Our calculation demonstrates that the gluon-fusion contribution to on-shell $W$-pair production only provides a $5 \%$ correction to the inclusive $W$-pair production cross section at the LHC. This result is consistent with analyses carried out for the processes $g g \rightarrow W Z, W \gamma$ and $Z \gamma[14,16]$. However, after taking into account realistic experimental cuts, the process $g g \rightarrow W^{*} W^{*} \rightarrow \ell \bar{\nu} \bar{\ell}^{\prime} \nu^{\prime}$ becomes sizeable and enhances the theoretical $W W$ background estimate for Higgs searches by about $30 \%$. In the following we will present some details of the calculation and a discussion of the numerical results.

\section{Calculation}

Our aim is to compute gluon-gluon induced off-shell $W$-boson pair production with decay into lepton-neutrino pairs:

$$
\begin{aligned}
g\left(p_{1}, \lambda_{1}\right)+g\left(p_{2}, \lambda_{2}\right) & \rightarrow W^{+*}\left(p_{3}\right)+W^{-*}\left(p_{4}\right) \\
& \rightarrow \bar{\ell}^{\prime}\left(p_{5},+\right)+\nu^{\prime}\left(p_{6},-\right)+\bar{\nu}\left(p_{7},+\right)+\ell\left(p_{8},-\right),
\end{aligned}
$$

where the momenta and helicities are given in brackets, and where $p_{3}=p_{5}+p_{6}$ and $p_{4}=p_{7}+p_{8}$. Generic Feynman diagram topologies are shown in Fig. 1. As mentioned in the introduction, we allow for arbitrary invariant masses of the $W$ bosons, but focus on the dominant contribution coming from massless intermediate quark loops. This simplifies the computation in two respects. First, the restricted number of scales allows for a compact representation of the amplitude, which has proven necessary to achieve a numerically stable evaluation. Second, in the limit of massless quark loops all triangle topologies in Fig. 1 with an intermediate photon or $Z$ boson vanish, and the box topologies form a gauge invariant set. Note that in the case of massive quarks in the loop the non-resonant terms are crucial for maintaining gauge invariance.

The amplitude $\mathcal{M}$ for the off-shell production of two charged vector bosons has the following structure:

$$
\mathcal{M}=\varepsilon_{1, \mu_{1}} \varepsilon_{2, \mu_{2}} \mathcal{M}^{\mu_{1} \mu_{2} \mu_{3} \mu_{4}} P_{\mu_{3} \nu_{3}}\left(p_{3}, M_{W}\right) P_{\mu_{4} \nu_{4}}\left(p_{4}, M_{W}\right) J_{3}^{\nu_{3}} J_{4}^{\nu_{4}}
$$


with the lepton currents and $W$ propagators given by

$$
\begin{aligned}
J_{3}^{\mu_{3}} & =\bar{u}\left(p_{6}\right) \gamma^{\mu_{3}} \frac{1}{2}\left(1-\gamma_{5}\right) v\left(p_{5}\right), \\
J_{4}^{\mu_{4}} & =\bar{u}\left(p_{8}\right) \gamma^{\mu_{4}} \frac{1}{2}\left(1-\gamma_{5}\right) v\left(p_{7}\right), \\
P^{\mu \nu}\left(p, M_{W}\right) & =\frac{g^{\mu \nu}}{p^{2}-M_{W}^{2}+i M_{W} \Gamma_{W}} .
\end{aligned}
$$

We have evaluated the Feynman diagrams using two independent methods. The expressions for the six box graphs have been compared with output from FeynArts 3.2 [17]. The algebraical manipulations were performed with FORM [18] and MAPLE.

Numerical tensor reduction which is commonly used in standard algebraic packages is in general not sufficient to achieve a numerically stable amplitude representation. In these approaches the problem of inverse Gram determinants is usually avoided by applying experimental cuts from the start such that kinematically dangerous regions do not contribute to the cross section. In the case of $W$-boson pair production with subsequent leptonic decays, however, this is not possible, as the invisible neutrinos do not allow to apply an experimental cut on the transverse momentum of the $W$ bosons to avoid large denominators. To overcome this problem we have performed a fully algebraic tensor and scalar integral reduction following the methods described in Ref. [19]. The result has been cross checked using standard methods [20, 21]. Each helicity amplitude has been grouped into explicitly gauge invariant tensor structures and expressed in a basis of scalar integrals similar to those defined in Ref. [22]. The respective coefficients have been simplified algebraically using MAPLE in a fully automated way so that at most one inverse power of a Gram determinant remained. Finally, the simplified helicity amplitudes were coded in two independent computer programs. Our representation of the cross section is numerically stable without any cuts. More details of the calculation will be presented in a forthcoming article.

\section{Results}

In this section we present numerical results for the process $p p \rightarrow W^{*} W^{*} \rightarrow \ell \bar{\nu} \bar{\ell}^{\prime} \nu^{\prime}$ at the LHC. We tabulate the total cross section and the cross section for two sets of experimental cuts. In addition, we show various differential distributions. The experimental cuts include a set of "standard cuts" [9], motivated by the finite acceptance and resolution of the detectors, where we require all charged leptons to be produced at $p_{T, \ell}>20 \mathrm{GeV}$ and $\left|\eta_{\ell}\right|<2.5$, and a missing transverse momentum $\not_{T}>25 \mathrm{GeV}$. Cross sections calculated with this set of cuts will be labelled $\sigma_{s t d}$.

As emphasized in the introduction, $W$-boson pair production constitutes the dominant irreducible background to Higgs searches in the $H \rightarrow W^{*} W^{*} \rightarrow \ell \bar{\nu} \bar{\ell}^{\prime} \nu^{\prime}$ decay mode, with a cross section times branching ratio more than five times larger than the Higgs-boson signal. ${ }^{2}$ Various cuts have been proposed for the experimental searches to enhance the signal-tobackground ratio $[1,2,23,24,25,26]$. We have adopted a set of cuts similar to those

\footnotetext{
${ }^{2}$ Other, less important backgrounds include $W$-boson production from top-quark decays.
} 
advocated in a recent experimental study [26]. In addition to the "standard cuts" defined above, we require that the opening angle between the two charged leptons in the plane transverse to the beam direction should satisfy $\Delta \phi_{T, \ell \ell}<45^{\circ}$ and that the dilepton invariant mass $M_{\ell \ell}$ be less than $35 \mathrm{GeV}$. Furthermore, the larger and smaller of the lepton transverse momenta are restricted as follows: $25 \mathrm{GeV}<p_{T, \text { min }}$ and $35 \mathrm{GeV}<p_{T, \max }<50 \mathrm{GeV}$. Finally, a jet-veto is imposed that removes events with jets where $p_{T \text {,jet }}>20 \mathrm{GeV}$ and $\left|\eta_{\text {jet }}\right|<3$. Cross sections evaluated with the Higgs selection cuts will be labelled $\sigma_{b k g}$.

To obtain numerical results we use the following set of input parameters:

$$
\begin{array}{lll}
M_{W}=80.419 \mathrm{GeV}, & M_{Z}=91.188 \mathrm{GeV}, & G_{\mu}=1.16639 \times 10^{-5} \mathrm{GeV}^{-2}, \\
\Gamma_{W}=2.06 \mathrm{GeV}, & \Gamma_{Z}=2.49 \mathrm{GeV}, & V_{\mathrm{CKM}}=\mathbb{1} .
\end{array}
$$

The weak mixing angle is given by $c_{\mathrm{w}}=M_{W} / M_{Z}, s_{\mathrm{w}}^{2}=1-c_{\mathrm{w}}^{2}$. The electromagnetic coupling is defined in the $G_{\mu}$ scheme as $\alpha_{G_{\mu}}=\sqrt{2} G_{\mu} M_{W}^{2} s_{\mathrm{w}}^{2} / \pi$. The masses of external fermions are neglected. We restrict our calculation to light intermediate fermions which are treated as massless. The $p p$ cross sections are calculated at $\sqrt{s}=14 \mathrm{TeV}$ employing the CTEQ6L1 and CTEQ6M [27] parton distribution functions at tree- and loop-level, corresponding to $\Lambda_{5}^{\mathrm{LO}}=165 \mathrm{MeV}$ and $\Lambda_{5}^{\overline{\mathrm{MS}}}=226 \mathrm{MeV}$ with one- and two-loop running for $\alpha_{s}(\mu)$, respectively. The renormalization and factorization scales are set to $M_{W}$. Fixedwidth Breit-Wigner propagators are used for unstable gauge bosons.

We compare results for $W W$ production in gluon scattering with LO and NLO results for the quark scattering processes. Since we are interested in $W W$ production as a background, the $g g \rightarrow H \rightarrow W W$ signal amplitude is not included. ${ }^{3}$ The LO and NLO quark scattering processes are computed with MCFM [10], which implements helicity amplitudes with full spin correlations [8] and includes finite-width effects and single-resonant corrections. Table 1 shows gluon and quark scattering cross sections for the LHC. Total cross sections $\left(\sigma_{t o t}\right)$ are compared with cross sections when standard LHC cuts $\left(\sigma_{s t d}\right)$ and selection cuts optimized for Higgs boson searches $\left(\sigma_{b k g}\right)$ are applied (see above for the definition of the cuts). The $g g$ process only yields a $5 \%$ correction to the total $W W$ cross section calculated from quark scattering at NLO QCD. When realistic Higgs search selection cuts are applied the correction increases to $30 \%$. We expect that the inclusion of the $t-b$ quark mediated $g g \rightarrow W W$ amplitude contribution will lead to a further increase. Note that the experimental Higgs search cuts include a jet veto which suppresses large contributions from gluon-quark scattering at NLO and thereby reduces the K-factor for $q \bar{q} \rightarrow W W$ from 1.6 to 1.1 .

Table 1 also includes an estimate of the remaining theoretical uncertainty due to the QCD scale variation. We have varied the renormalization and factorization scales independently between $M_{W} / 2 \leq \mu_{\mathrm{ren}, \mathrm{fac}} \leq 2 M_{W}$. We find that the largest cross section prediction corresponds to choosing $\mu_{\mathrm{ren}}=M_{W} / 2$ and $\mu_{\mathrm{fac}}=2 M_{W}$, while the reverse combination $\mu_{\text {ren }}=2 M_{W}$ and $\mu_{\mathrm{fac}}=M_{W} / 2$ yields the smallest value. For the gluon-gluon induced contribution this is obvious, as the renormalization and factorization scale dependence is

\footnotetext{
${ }^{3}$ In the case of a light, narrow-width Higgs boson, interference effects between signal and background are expected to be strongly suppressed, see Refs. [28, 29].
} 
entirely due to $\alpha_{\mathrm{s}}$ and the parton distribution functions, respectively: decreasing $\mu_{\text {ren }}$ increases $\alpha_{\mathrm{s}}$, while increasing $\mu_{\mathrm{fac}}$ increases the gluon-gluon luminosity. The latter effect turns out to be small compared to the former. For the $g g \rightarrow W W$ process we find a scale uncertainty of approximately 25\%. The scale dependence of the NLO quark-scattering contribution is more intricate and depends sensitively on the cuts employed. For the $q \bar{q} \rightarrow W W$ process we find approximately $5 \%$ uncertainty. The Higgs selection cuts, which suppress the gluon-quark scattering contribution, further reduce the scale dependence to a level of only about $2 \%$. This is a remarkably small change that, we suspect, underestimates the uncertainty. A more detailed discussion of the scale uncertainty for $q \bar{q} \rightarrow W W$ can be found in Ref. [9].

The cross section for $p p \rightarrow W W \rightarrow \ell \bar{\nu} \bar{\ell}^{\prime} \nu^{\prime}$ is proportional to the fourth power of the electromagnetic coupling $\alpha$. As mentioned above, we have evaluated $\alpha$ in the $G_{\mu}$ scheme. The $G_{\mu}$ scheme is a preferred choice as it absorbs large universal higher-order electroweak effects in the LO prediction. Alternatively, $\alpha$ can be identified with the finestructure constant $\alpha(0)$ or the running electromagnetic coupling $\alpha\left(Q^{2}\right)$ at a high energy scale $Q$. Setting $\alpha=\alpha(0)\left[\alpha=\alpha\left(M_{Z}\right)\right]$ results in a decrease [increase] of the cross-section prediction by approximately $10 \%$.

Off-shell effects reduce the total $g g \rightarrow W W$ cross section in narrow width approximation by $2.6 \%$. The Higgs search selection cuts amplify the effect to about $-6 \%$. The quark scattering process is affected in a similar way. We conclude that the narrow width approximation yields conservative estimates for the $p p \rightarrow W W$ background. Note that the Higgs search cuts have been optimized for a Higgs boson mass of $M_{\text {Higgs }}=165 \mathrm{GeV}$. Off-shell effects may have a larger impact when other cuts, more suitable for lighter Higgs masses $M_{\text {Higgs }}<2 M_{W}$, are applied.

We have compared our results with the $g g \rightarrow W W$ cross section calculation presented in Ref. [12]. Adopting the set-up of Ref. [12] we find perfect agreement for the invariant $W W$ mass distribution. Note that the large impact of the gluon-gluon induced contributions to $W$-pair production at the LHC suggested in previous analyses $[12,13]$ is not confirmed in our calculation. The difference is due to using modern parton distribution functions, which show a less pronounced rise of the gluon-luminosity towards small values of $x$, and the associated smaller values of the strong coupling $\alpha_{\mathrm{s}}$.

Selected differential distributions for $p p \rightarrow W^{*} W^{*} \rightarrow \ell \bar{\nu} \bar{\ell}^{\prime} \nu^{\prime}$ at the LHC are shown in Figs. 2, 3 and 4. The standard set of cuts defined above has been applied throughout.

Figure 2 shows the distribution in the invariant mass of the pair of charged leptons. We compare the gluon-gluon induced contribution with the quark scattering process in LO and NLO. We observe that the invariant mass distribution of the gluon-gluon induced process is similar in shape to the quark scattering contributions and suppressed by more than one order of magnitude in normalization.

$W$-boson pairs produced in quark-antiquark scattering at the LHC are in general strongly boosted along the beam axis. Gluon induced processes on the other hand result in $W W$ events at more central rapidities. This feature is born out by the distribution in the pseudorapidity of the negatively charged lepton shown in Fig. 3. In order to distinguish the shapes of the various contributions we have chosen a linear vertical scale and plot 
the gluon-gluon contribution multiplied by a factor 10. Compared to LO quark-antiquark scattering, the lepton distribution of the gluon-gluon process shows a more pronounced peak at central rapidities. We also observe an enhancement of the NLO corrections at central rapidities which is due to the substantial contribution of gluon-quark processes at NLO.

Figure 4 finally shows the distribution in the transverse-plane opening angle of the charged leptons. This observable reflects the spin correlations between the $W W$ pair and allows one to discriminate $W$ bosons originating from scalar Higgs decays and $W W$ continuum production [30]. Note that the importance of the gluon-gluon process is enhanced by the Higgs search selection cuts which require a small opening angle $\Delta \phi_{T, \ell \ell}<45^{\circ}$.

\section{Conclusions}

We have calculated the loop-induced gluon-fusion process $g g \rightarrow W^{*} W^{*} \rightarrow \ell \bar{\nu} \bar{\ell}^{\prime} \nu^{\prime}$ which provides an important background for Higgs boson searches in the $H \rightarrow W W$ channel at the LHC. We have presented numerical results for the total cross section, the cross section with two sets of experimental cuts and various differential distributions. Our calculation demonstrates that the gluon-fusion contribution to on-shell $W$-pair production only yields a $5 \%$ correction to the inclusive $W$-pair production cross section at the LHC. However, after imposing realistic Higgs search selection cuts, the process $g g \rightarrow W^{*} W^{*} \rightarrow \ell \bar{\nu} \bar{\ell}^{\prime} \nu^{\prime}$ becomes the dominant higher-order correction to the $W W$ background estimate and enhances the theoretical prediction from quark-antiquark scattering at NLO by approximately $30 \%$. We conclude that gluon-gluon induced $W$-pair production is essential for a reliable description of the background and has to be taken into account to exploit the discovery potential of Higgs boson searches in the $p p \rightarrow H \rightarrow W W \rightarrow$ leptons channel at the LHC.

\section{Acknowledgments}

A major part of this work was done when all authors were at the University of Edinburgh. We would like to thank the Edinburgh Particle Physics Theory Group and the British Research Council PPARC for support. We are grateful to Herbi Dreiner for drawing our attention to the importance of gluon-induced backgrounds to Higgs boson searches. We benefited from discussions with Michael Dittmar, Herbi Dreiner, and Adrian Signer. Special thanks go to Peter Marquard and Jochum van der Bij for a comparison of results and to John Campbell for his help in using the MCFM program. The work of T.B. is supported by the Bundesministerium für Bildung und Forschung (BMBF, Bonn, Germany) under the contract number 05HT4WWA2. The work of N.K. is supported in part by the DFG Sonderforschungsbereich/Transregio 9 "Computer-gestützte Theoretische Teilchenphysik".

\section{References}

[1] M. Dittmar and H. K. Dreiner, Phys. Rev. D 55 (1997) 167 [arXiv:hep-ph/9608317].

[2] M. Dittmar and H. K. Dreiner, "h0 $\rightarrow \mathrm{W}+\mathrm{W}-\rightarrow \mathrm{l}+\mathrm{l}$ '- nu/l anti-nu/l' as the dominant SM Higgs search mode at the LHC for $\mathrm{M}(\mathrm{h} 0)=155-\mathrm{GeV}$ to 180-GeV," arXiv:hep-ph/9703401, 
published in the proceedings of the Ringberg Workshop "The Higgs Puzzle - What can We Learn from LEP2, LHC, NLC, and FMC?", 8-13 December 1996, Ringberg, Germany. Editor B.A. Kniehl, Singapore, World Scientific, 1997.

[3] N. Kauer, T. Plehn, D. L. Rainwater and D. Zeppenfeld, Phys. Lett. B 503 (2001) 113 [arXiv:hep-ph/0012351].

[4] S. Haywood et al., "Electroweak physics," arXiv:hep-ph/0003275, published in the proceedings of the "CERN Workshop on Standard Model Physics (and more) at the LHC", 14-15 October 1999, Geneva, Switzerland. Editors G. Altarelli and M.L. Mangano, Geneva, CERN, 2000.

[5] J. Ohnemus, Phys. Rev. D 44 (1991) 1403.

[6] S. Frixione, Nucl. Phys. B 410 (1993) 280.

[7] J. Ohnemus, Phys. Rev. D 50 (1994) 1931 [arXiv:hep-ph/9403331].

[8] L. J. Dixon, Z. Kunszt and A. Signer, Nucl. Phys. B 531 (1998) 3 [arXiv:hep-ph/9803250].

[9] L. J. Dixon, Z. Kunszt and A. Signer, Phys. Rev. D 60 (1999) 114037 [arXiv:hep-ph/9907305].

[10] J. M. Campbell and R. K. Ellis, Phys. Rev. D 60 (1999) 113006 [arXiv:hep-ph/9905386].

[11] E. Accomando, A. Denner and A. Kaiser, Nucl. Phys. B 706 (2005) 325 [arXiv:hep-ph/0409247].

[12] E. W. N. Glover and J. J. van der Bij, Phys. Lett. B 219 (1989) 488.

[13] C. Kao and D. A. Dicus, Phys. Rev. D 43 (1991) 1555.

[14] K. L. Adamson, D. de Florian and A. Signer, Phys. Rev. D 65 (2002) 094041 [arXiv:hep-ph/0202132].

[15] C. Zecher, T. Matsuura and J. J. van der Bij, Z. Phys. C 64 (1994) 219 [arXiv:hep-ph/9404295].

[16] K. L. Adamson, D. de Florian and A. Signer, Phys. Rev. D 67 (2003) 034016 [arXiv:hep-ph/0211295].

[17] T. Hahn, Comput. Phys. Commun. 140 (2001) 418 [hep-ph/0012260].

[18] J. A. M. Vermaseren, arXiv:math-ph/0010025 (unpublished).

[19] T. Binoth, J. P. Guillet and G. Heinrich, Nucl. Phys. B 572 (2000) 361 [arXiv:hep-ph/9911342].

[20] G. 't Hooft and M. J. G. Veltman, Nucl. Phys. B 153 (1979) 365.

[21] G. Passarino and M. J. G. Veltman, Nucl. Phys. B 160 (1979) 151.

[22] T. Binoth, J. P. Guillet and F. Mahmoudi, JHEP 0402 (2004) 057 [arXiv:hep-ph/0312334].

[23] M. Dittmar and H. K. Dreiner, CMS-NOTE-1997-083 (unpublished).

[24] K. Jakobs, T. Trefzger, ALTLAS-PHYS-2000-015 (unpublished).

[25] D. Green, K. Maeshima, J. Marraffino, R. Vidal, J. Womersley, W. Wu and S. Kunori, J. Phys. G 26 (2000) 1751. 
[26] G. Davatz, G. Dissertori, M. Dittmar, M. Grazzini and F. Pauss, JHEP 0405 (2004) 009 [arXiv:hep-ph/0402218].

[27] J. Pumplin, D. R. Stump, J. Huston, H. L. Lai, P. Nadolsky and W. K. Tung, JHEP 0207 (2002) 012 [arXiv:hep-ph/0201195].

[28] D. A. Dicus and S. S. D. Willenbrock, Phys. Rev. D 37 (1988) 1801.

[29] L. J. Dixon and M. S. Siu, Phys. Rev. Lett. 90 (2003) 252001 [arXiv:hep-ph/0302233].

[30] C. A. Nelson, Phys. Rev. D 37 (1988) 1220. 

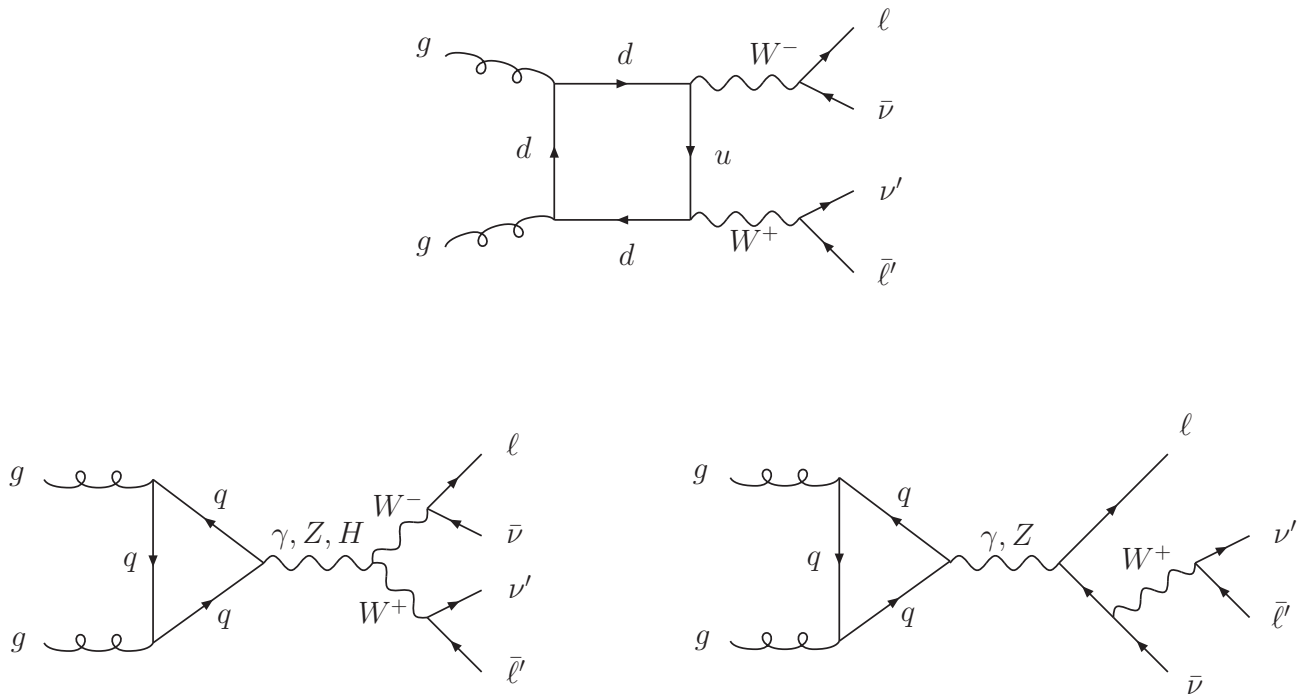

Figure 1: Generic Feynman diagrams for the process $g g \rightarrow W^{*} W^{*} \rightarrow \ell \bar{\nu} \bar{\ell}^{\prime} \nu^{\prime}$.

\begin{tabular}{|c|c|cc|c|c|}
\cline { 2 - 5 } \multicolumn{1}{c|}{} & \multicolumn{5}{|c|}{$\sigma\left(p p \rightarrow W^{*} W^{*} \rightarrow \ell \bar{\nu} \bar{\ell}^{\prime} \nu^{\prime}\right)[\mathrm{fb}]$} \\
\cline { 2 - 5 } \multicolumn{1}{c|}{$g g$} & $\frac{\sigma_{\mathrm{NLO}}}{\sigma_{\mathrm{LO}}}$ & $\frac{\sigma_{\mathrm{NLO}+g g}}{\sigma_{\mathrm{NLO}}}$ \\
\cline { 2 - 6 } & & $\mathrm{LO}$ & $\mathrm{NLO}$ & 1.57 & 1.04 \\
\hline$\sigma_{\text {tot }}$ & $53.61(2)_{-10.8}^{+14.0}$ & $875.8(1)_{-67.5}^{+54.9}$ & $1373(1)_{-79}^{+71}$ & 1.57 & 1.05 \\
\hline$\sigma_{s t d}$ & $25.89(1)_{-5.29}^{+6.85}$ & $270.5(1)_{-23.8}^{+20.0}$ & $491.8(1)_{-32.7}^{+27.5}$ & 1.82 & 1.29 \\
\hline$\sigma_{b k g}$ & $1.385(1)_{-0.31}^{+0.40}$ & $4.583(2)_{-0.48}^{+0.42}$ & $4.79(3)_{-0.13}^{+0.01}$ & 1.05 & 1.05 \\
\hline
\end{tabular}

Table 1: Cross sections for the gluon and quark scattering contributions to $p p \rightarrow W^{*} W^{*} \rightarrow \ell \bar{\nu} \bar{\ell}^{\prime} \nu^{\prime}$ at the LHC $(\sqrt{s}=14 \mathrm{TeV})$ without selection cuts $($ tot $)$, with standard LHC cuts $\left(s t d: p_{T, \ell}>20\right.$ $\left.\mathrm{GeV},\left|\eta_{\ell}\right|<2.5, p_{T}>25 \mathrm{GeV}\right)$ and Higgs search selection cuts (bkg, see main text) applied. The integration error is given in brackets. The errors denote the QCD scale uncertainty obtained by varying renormalization and factorization scales independently between $M_{W} / 2 \leq \mu_{\text {ren,fac }} \leq 2 M_{W}$. We also show the ratio of the NLO to LO cross sections and the ratio of the combined $\mathrm{NLO}+g g$ contribution to the NLO cross section at the central scale $\mu=M_{W}$. 


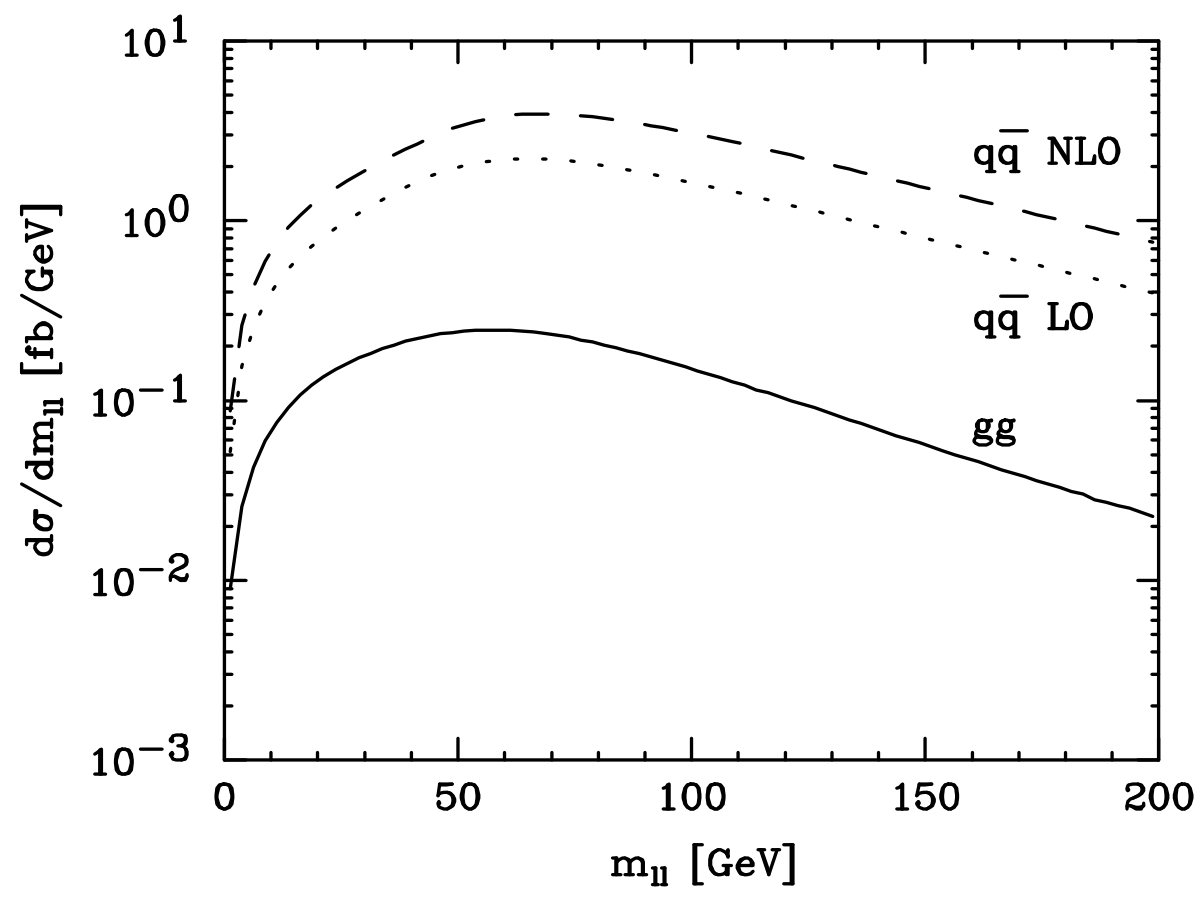

Figure 2: Distributions in the charged lepton-pair invariant mass $m_{\ell \ell}$ for the gluon scattering process (solid) and the quark scattering process in LO (dotted) and NLO QCD (dashed) of $p p \rightarrow$ $W^{*} W^{*} \rightarrow \ell \bar{\nu} \bar{\ell}^{\prime} \nu^{\prime}$ at the LHC. Input parameters as defined in the main text. Standard LHC cuts have been applied (see main text and Table 1).

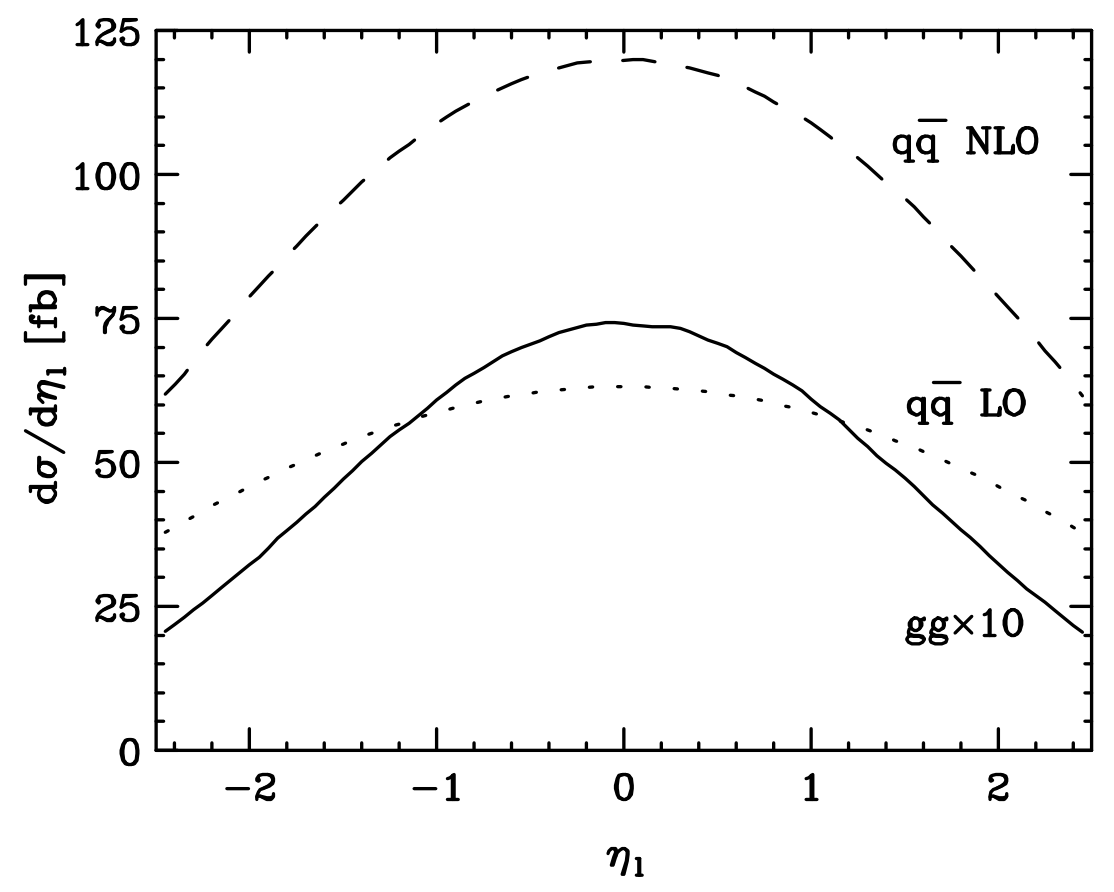

Figure 3: Distributions in the pseudorapidity $\eta_{\ell^{-}}$of the negatively charged lepton. Details as in Fig. 2. The $g g$ distribution is displayed after multiplication with a factor 10 . 


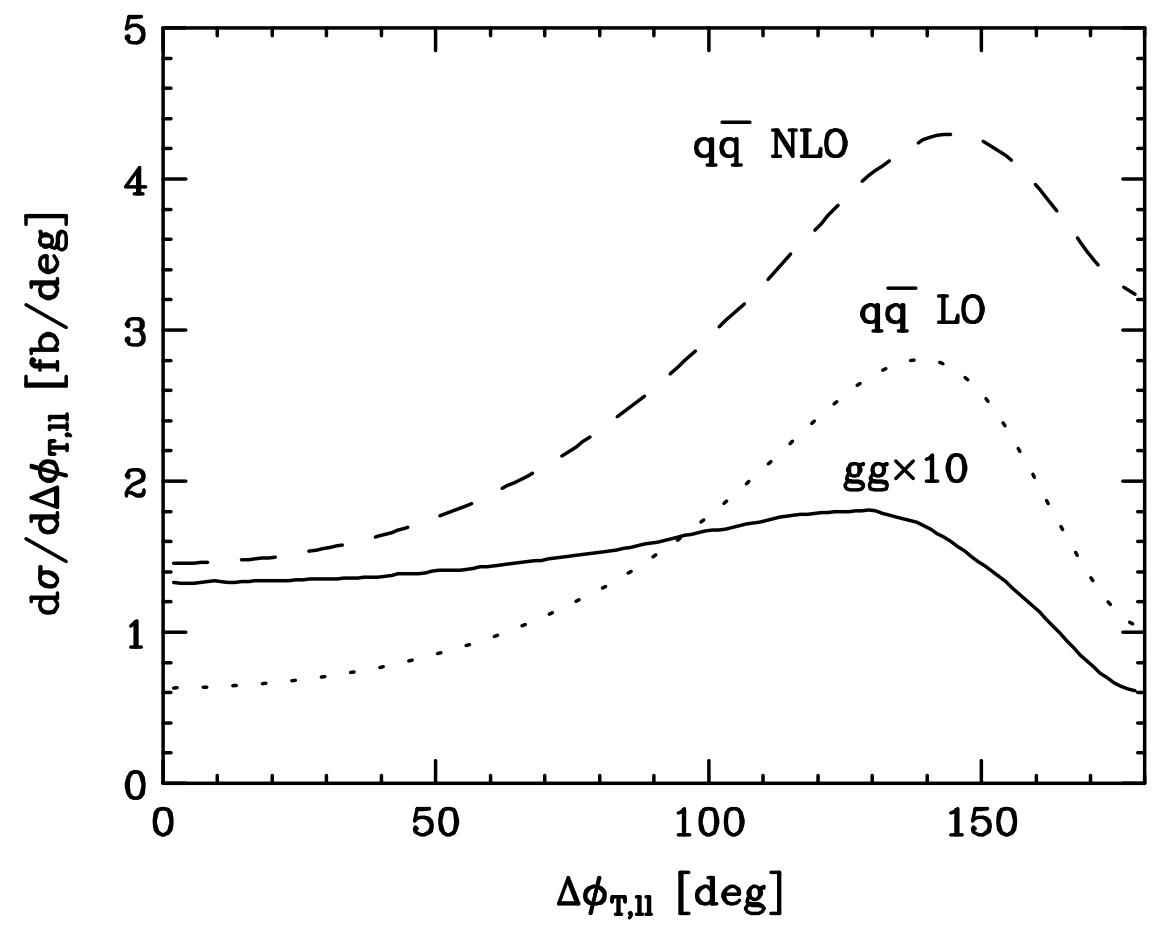

Figure 4: Distributions in the transverse-plane opening angle of the charged leptons $\Delta \phi_{T, \ell \ell}$. Details as in Fig. 2. The $g g$ distribution is displayed after multiplication with a factor 10 . 